\title{
UTILIZAÇÃO DA FARINHA DE ALGAS CALCÁREAS NA ALIMENTAÇÃO ANIMAL
}

\author{
USE OF SEAWED FLOUR IN THE ANIMAL FEEDING
}

\begin{abstract}
Melo, T.V. ${ }^{1}$ e A. M. A. Moura ${ }^{2}$
'Universidade Estadual Paulista Júlio de Mesquita Filho. Campus de Jaboticabal. Faculdade de Ciências Agrárias e Veterinárias. thiagovmelo@gmail.com

${ }^{2} Z$ ootecnista. Centro de Pesquisas Aggeu Magalhães. CPqAM-Fiocruz. Campus da UFPE, Várzea. Av. Moraes Rego, s/n. Caixa Postal 7472. CEP 50670-420. Recife-PE. Brasil. antoniol@cpqam.fiocruz.br
\end{abstract}

\section{PalaVRas chave adicionais}

Fonte de cálcio. Biodisponibilidade. Alga marinha. Lithothamnium calcareum.

\section{RESUMO}

O cálcio é um elemento essencial, tendo função básica na formação dos ossos e dentes, e em diversos processos fisiológicos. Na alimentação animal são utilizadas diversas fontes de cálcio, dentre elas o cálcio proveniente das algas marinhas calcáreas. Sabe-se que as algas calcáreas retém elevado índice de elementos minerais do meio marinho, além de apreciável quantidade de substancias nutritivas. O Lithothamnium calcareum pertence ao grupo das algas vermelhas ou rodofíceas, da família das Coralineacea, é uma alga de aspecto calcário, pois absorve o carbonato de cálcio e magnésio.

\section{SUMMARY}

Calcium is an essential element, it has basic function in bones and teeth synthesis, yet in various physiological processes. In the animal feeding they are used several sources of calcium, including calcium from calcareous seaweed. It is well-known that the calcareous algae retain high levels of mineral elements of the marine environment, and considerable amount of nutrients. The Lithothamnium calcareum belongs to the group of red algae or rhodophytes, the family of Coralineacea are calcareous algae in appearance, because it absorbs the calcium carbonate and magnesium.

\section{AdDitional KEYWORDS}

Source of calcium. Bioavailability. Seaweed. Lithothamnium calcareum.

\section{INTRODUÇÃO}

O cálcio é um elemento químico presente nos fluidos e tecidos corporais, sendo essencial na formação dos ossos, dentes e em diversos processos fisiológicos (Macari et al., 2002). As fontes de cálcio podem ser de origem inorgânica (rochas) ou orgânica (farinha de ossos, conchas, e algas). Geralmente as fontes de cálcio utilizadas na alimentação animal são oriundas de rochas, como o calcário e o fosfato bicálcico, pois, são mais abundantes e de menor custo.

A solubilidade das fontes de cálcio é um fator indicativo na qualidade, já que apresenta alta correlação com a biodisponibilidade e absorção intestinal do cálcio. As fontes de cálcio de origem orgânica, como a farinha de ostras e farinha de casca de ovos são fontes de maior solubilidade em relação as fontes de rochas (Melo et al., 2006).

Porém, as fontes inorgânicas de cálcio, são recursos minerais não-renováveis e sua extração promove importante impacto ambiental. O fosfato bicálcico por se tratar de uma fonte mista de cálcio e fósforo, apresenta custo elevado, ao contrário do 


\section{MELO E MOURA}

calcário que dentre os ingredientes da ração é o que apresenta menor custo por quilograma.

Os alimentos de origem vegetal, normalmente milho e soja, constituem a base da alimentação de aves e possuem teores de cálcio em níveis insuficientes para suprir as exigências nutricionais. Desta forma, há necessidade de fazer uma suplementação de cálcio na dieta para atender estas exigências dos animais (Muniz et al., 2007).

$O$ cálcio, o fósforo e a vitamina $D$ são elementos intimamente associados no metabolismo animal, muitas vezes combinados entre si, de modo que a carência de um deles na dieta limita o desempenho das aves (Macari et.al., 2002). Assim, a biodisponibilidade das fontes de cálcio influenciam no nível de suplementação.

No entanto, o cálcio em excesso pode agir como antagonista dificultando a absorção de alguns minerais tais como ferro, cobre, zinco, magnésio, sódio, potássio, entre outros (Waldroup, 1996). O Magnésio substitui parte do cálcio, resultando em cristais mais densos e menos solúveis, além de ser antagônico ao cálcio, podendo influenciar o mecanismo de absorção intestinal.
Suplementos contendo microminerais complexados a moléculas orgânicas têm sido desenvolvidos industrialmente com base na teoria da maior biodisponibilidade que a de fontes inorgânicas (Spears, 1996). Preconiza-se que minerais orgânicos têm maior solubilidade, estrutura química estável e natureza eletricamente neutra no trato digestivo. Logo, estes não participariam de reações que poderiam transformar o íon metálico livre em complexos insolúveis indesejáveis. Foi observado que a substituição crescente de fonte inorgânica de minerais por um suplemento contendo minerais orgânicos aumentou o consumo de matéria seca e a digestibilidade em bovinos consumindo dietas de baixo valor nutritivo (Langwinski e Ospina, 2001).

As buscas de novas alternativas que não sejam derivadas de rochas, de maior biodisponibilidade, são de extrema importância para se maximizar o desempenho animal e minimizar custos. Segundo Fassani et al. (2004), a falta de conhecimento das características fisico-químicas dos calcários pode ocasionar variação na atenção das exigências nutricionais obtidas em pesquisas científicas, o que leva muitos nutricionistas à utilização de altos níveis de

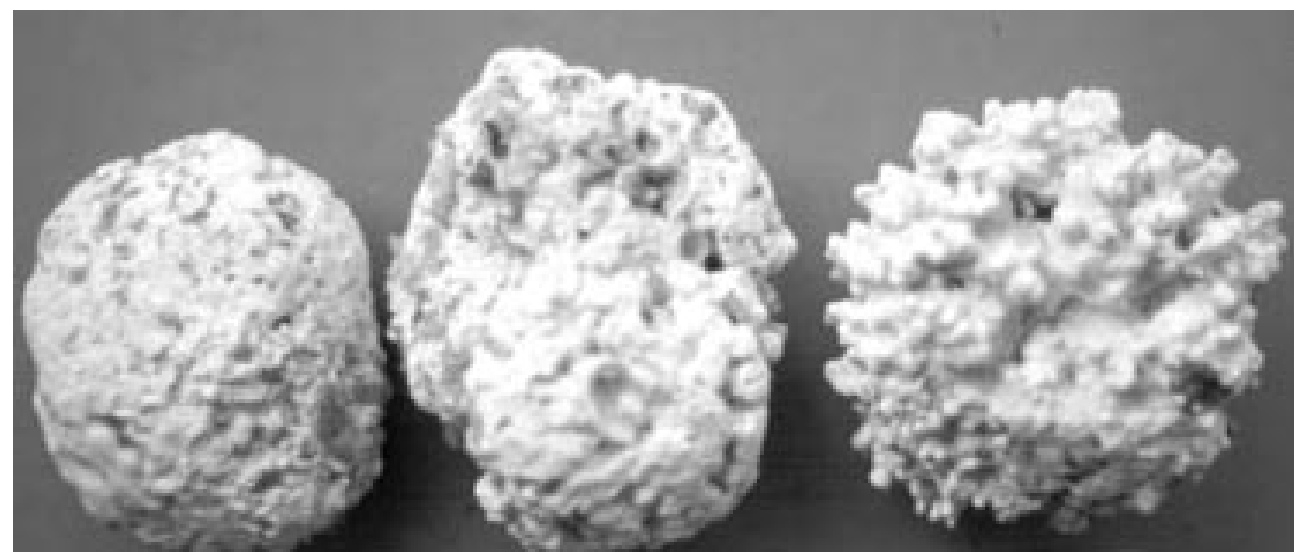

Figura 1. Foto ilustrativa da alga calcárea Lithothamnium calcareum (Algarea, 2008). (Photo illustration of calcareous algae Lithothamnium calcareum (Algarea, 2008)). 


\section{UTILIZAÇÃO DA FARINHA DE ALGAS CALCÁREAS NA ALIMENTAÇÃO ANIMAL}

cálcio em rações comerciais. Uma destas novas alternativas é o, fonte de cálcio proveniente das algas calcáreas.

Sabe-se que as algas marinhas calcáreas retêm elevado índice de elementos minerais do meio marinho, além de apreciável quantidade de substancias nutritivas. O Lithothamnium calacareum (figura 1) pertence ao grupo das algas vermelhas ou rodofíceas, da família das coralináceas. É uma alga de aspecto calcário, pois absorve o carbonato de cálcio e magnésio. Não é fonte de proteína, vitaminas, carboidratos e lipídeos, somente de macro e micro minerais (tabela I) em concentrações variadas, dependendo do local, estação do ano e profundidade.

A semelhança entre o calcário de origem continental e as algas Lithothamnium se limita basicamente a alguns elementos químicos comuns a ambos.

As algas marinhas calcáreas são as plantas que crescem naturalmente no meio marinho e em profundidades das mais variadas. A renovação é permanente, contanto que haja incidência de luz natural, se tornando uma fonte de macro e microminerais renovável. O produto pode ser aplicado no estado natural ou após secagem e moagem.

Contudo, comprovada sua viabilidade zootécnica, a opção entre as diferentes fontes é realizada com base no custo por unidade de fósforo biodisponível, e não na unidade de fósforo total (Couto et al., 2008).

Esta revisão, aborda a utilização da farinha de algas calcáreas Lithothamnium calcareum na alimentação animal, pois diante deste tema, ainda são incipientes os

Tabela I . Principais nutrientes da farinha de algas marinhas (Lithothamnium calcareum) Análise típica*. (Key nutrients of seaweed flour (Lithothamnium calcareum) - Typical analysis*).

\begin{tabular}{|c|c|c|c|}
\hline Cálcio (Ca) & $32,5 \%$ & Magnésio (Mg) & $2,0 \%$ \\
\hline Silício (Si) & $0,95 \%$ & Boro (B) & 20 ppm \\
\hline Cobre (Cu) & $2 \mathrm{ppm}$ & Enxofre (S) & $0,50 \%$ \\
\hline Ferro (Fe) & $0,25 \%$ & Manganês (Mn) & 20 ppm \\
\hline Molibdênio (Mo) & $5 \mathrm{ppm}$ & Zinco (Zn) & 11 ppm \\
\hline Cromo (Cr) & $4 \mathrm{ppm}$ & Cobalto (Co) & $5 \mathrm{ppm}$ \\
\hline Cloro (Cl) & $0,20 \%$ & Fósforo $(\mathrm{P})$ & $0,03 \%$ \\
\hline Potássio (K) & $0,01 \%$ & Sódio (Na) & $0,26 \%$ \\
\hline Vanádio (V) & $4 \mathrm{ppm}$ & Níquel (Ni) & 10 ppm \\
\hline lodo (I) & 12 ppm & Selênio (Se) & $1 \mathrm{ppm}$ \\
\hline Flúor (F) & 800 ppm & Antimônio (Sb) & $<1 \mathrm{ppm}$ \\
\hline Prata $(\mathrm{Ag})$ & $<1$ ppm & Alumínio (AL) & $0,7 \%$ \\
\hline Bário (Ba) & 15 ppm & Berílio (Be) & $<1 \mathrm{ppm}$ \\
\hline Bismuto (Bi) & $8 \mathrm{ppm}$ & Cádmio (Cd) & $<1 \mathrm{ppm}$ \\
\hline Lantânio (La) & $8 \mathrm{ppm}$ & Lítio (Li) & $5 \mathrm{ppm}$ \\
\hline Cádmio (Cd) & $<1$ ppm & Escândio (Sc) & $<1 \mathrm{ppm}$ \\
\hline Titânio (Ti) & $0,01 \%$ & Estanho (Sn) & $<10 \mathrm{ppm}$ \\
\hline Tungstênio (W) & $<10$ ppm & Ítrio $(\mathrm{Y})$ & $5 \mathrm{ppm}$ \\
\hline Zircônio (Zr) & $5 \mathrm{ppm}$ & Arsênio (As) & $4 \mathrm{ppm}$ \\
\hline Cério (Ce) & $8 \mathrm{ppm}$ & Neodímio (Nd) & $4 \mathrm{ppm}$ \\
\hline Samário (SM) & 0,5 ppm & Európio (Eu) & $0,5 \mathrm{ppm}$ \\
\hline Gadolínio (Gd) & $0,05 \mathrm{ppm}$ & Disprósio (Dy) & $0,3 \mathrm{ppm}$ \\
\hline Hólmio (Ho) & $0,05 \mathrm{ppm}$ & Érbio (Er) & $0,2 \mathrm{ppm}$ \\
\hline Itérbio (Yb) & 0,2 ppm & Lutécio (Lu) & $0,05 \mathrm{ppm}$ \\
\hline
\end{tabular}

* Sendo um produto natural os teores acima podem variar (Melo, 2006) 


\section{MELO E MOURA}

resultados sobre a utilização deste suplemento na alimentação animal.

\section{HISTÓRIA E APLICAÇÃO DAS ALGAS CALCÁREAS}

De acordo com Cressard (1991), citado por Dias (2000), a utilização de materiais marinhos para uso agrícola parece muito antiga. Pline em sua "Histoire Naturele" diz que a Bretanha e os gauleses inventaram uma arte de fertilizar o solo por meio de uma certa terre marga. Candem, em sua obra Britannia no inicio do século XVII escreveu que "o solo do Condado de Devonshire seria quase estéril se não fosse melhorado por um tipo de areia que se retira do mar e que o torna muito fértil, se impregnando de alguma forma na terra e por esta razão esta areia se compra muito caro nos lugares mais afastados da costa".

A indústria de utilização do Lithothamnium iniciou-se e desenvolveu-se na França a partir do final da Segunda Guerra Mundial. Foi inicialmente utilizada somente como fertilizante, e a empresa pioneira foi a Timac da França, que em 1956 vendia o fertilizante NPK diferenciando dos demais com a adição da farinha de algas calcáreas Lithothamnium, tornando-se assim, o segundo maior fabricante de fertilizantes da Europa e o primeiro da França. Cabioch (1970) cita que desde a antigüidade, as algas calcáreas vem sendo utilizadas na Europa para tratamento de solos ácidos.

Além da França, a Irlanda (Bosence, 1976) e Inglaterra começaram a produzir para consumo interno, pequenas quantidades voltadas principalmente para a complementação da ração animal, o mesmo acontecendo no Japão. No Brasil, com exceção do petróleo, a explotação de recursos minerais marinhos tem sido pontual e descontínua, restringindo-se à extração de areias para regeneração de praias e extração localizada de conchas e algas calcárias nos estados do Rio de Janeiro e Espírito Santo (Gomes, et al., 2000). A utilização do
Lithothamnium no Brasil se restringia somente na agricultura, e nos últimos anos com o lançamento de produtos a base de Lithothamnium calcareum como suplemento em rações para animais, que este vem despertando o interesse de pesquisas de instituições públicas e privadas. A alga calcária é extraída do seu meio por processos manuais e mecânicos, e a matéria prima "in natura" é lavada, desidratada e moída, e em seguida ensacada.

\section{A UTILIZAÇÃO DA FARINHA DE ALGAS CALCÁREAS NA PRODUÇÃO ANIMAL}

Na produção animal, onde a alimentação representa cerca de $70 \%$ do custo de produção, o suprimento de cálcio participa com expressiva proporção nas formulações comerciais e a utilização de complementos alternativos destes minerais, quando disponíveis na região, se torna ferramenta importante para maximização do lucro líquido para o produtor.

Como fonte alternativa de cálcio, pode ser utilizado a farinha de algas calcáreas (Lithothamnium calcareum). De acordo com Dias (2000), as algas calcárias são compostas basicamente por carbonato de cálcio e magnésio, além de conter mais de 20 oligoelementos, presentes em quantidades variáveis, tais como $\mathrm{Fe}, \mathrm{Mn}, \mathrm{B}, \mathrm{Ni}, \mathrm{Cu}, \mathrm{Zn}$, $\mathrm{Mo}$, Se e Sr. O produto pode ser aplicado no estado natural ou após secagem e moagem. As principais características que potencializam a atuação deste produto são atribuídas à maior disponibilidade dos micronutrientes que se encontram adsorvidos nas paredes celulares, sendo assim facilmente assimiláveis pelas plantas e animais e à elevada porosidade das algas $(>40 \%)$ que propicia maior superfície específica de atuação.

\section{AUTILIZAÇÃONA ALIMENTAÇÃODE EQUINOS}

Os eqüinos são animais considerados atletas, e segundo Berberian and Lenci(1983) 


\section{UTILIZAÇÃO DA FARINHA DE ALGAS CALCÁREAS NA ALIMENTAÇÃO ANIMAL}

têm-se observado excelentes cavalos que tiveram uma campanha muito curta por apresentarem alterações graves dos ossos e tendões.

Segundo Berberian and Lenci (1983), a utilização do Lithothamnium calcareum como suplemento mineral na alimentação de cavalos P.S.I. revelou-se um excelente corretivo mineral e orgânico, melhorando e aumentando a disponibilidade biológica dos nutrientes existentes na ração.

Os mesmos autores Lenci and Berberian (1984) estudaram cinco casos de fraturas ósseas em cavalos P.S.I. de corrida no Jockey Club de São Paulo-Brasil, (onde a recomendação veterinária inicial apontava a cirurgia como melhor solução), foram adicionados 10 gramas duas vezes ao dia da farinha de algas calcáreas Lithothamnium calcareum na ração, com cada caso acompanhado com a utilização do raio $\mathrm{X}$ e observaram que após trinta dias, as fissuras consolidaram-se. Depois de sessenta dias tendo prosseguido a terapia, os animais já se encontravam em condições de retornarem gradativamente às pistas.

\section{A UTILIZAÇÃONA ALIMENTAÇÃODE AVES E SUÍNOS}

Na alimentação de aves e suínos o cálcio possue um importante papel, principalmente para poedeiras, pois necessitam de grande concentração de cálcio disponível para formação da casca dos ovos e também frangos de corte, por possuírem uma alta taxa de crescimento em pouco tempo, acarretando problemas na formação dos ossos, principalmente displasia tibial.

Pelícia et al. (2006) estudaram o efeito da combinação de fontes de cálcio sobre o desempenho e qualidade dos ovos de poedeiras comerciais, e concluíram que é possível a inclusão de cálcio marinho Lithothamnium na dieta de poedeiras em até $45 \%$ de substituição do calcário calcítico, sem que ocorram prejuízos ao desempenho e a qualidade dos ovos, desde que econo- micamente viável.

Trabalhando com codornas japonesas (Coturnix japonica) Perali et al. (2003) referenciaram aumento na produção de ovos em 4,16 pontos percentuais em relação à testemunha na adição de $0,25 \%$ deste produto. Porém o aumento na produção de ovos não foi observado por Melo et al. (2008a; 2008b), os quais avaliaram a utilização da farinha de algas calcáreas Lithothamnium calcareum no desempenho e qualidade de ovos de codornas japonesas, e observaram que o suplemento mostrou evidencias de melhoria na casca dos ovos e um aumento significativo no peso da gema, porém as características de desempenho não foram influenciadas pela utilização da farinha de algas calcáreas.

Em frangos de corte, nos estudos realizados por Zanini et al. (2000a), utilizando farinha de algas calcáreas como fonte de cálcio na ração de frangos de corte, concluíram que o uso de farinha de algas pode substituir totalmente o calcário sem prejudicar o desempenho dos animais, porém devemos nos atentar sobre o custo final desta substituição. De acordo com Airhart et al. (2002), o cálcio proveniente do Lithothamnium calcareum apresentou maior biodisponibilidade do que aquele proveniente do calcário, resultando em melhor conversão alimentar em frangos de corte. Efeitos de melhora na conversão alimentar também foram observados por Pope et al. (2002), em frangos de corte suplementados com Lithothamnium calcareum, os quais apresentaram maior ganho de peso e melhor rendimento de peito, devido a melhora $n$ conversão alimentar.

Estes resultados de melhora n conversão alimentar podem estar relacionadas à maior solubilidade do cálcio proveniente Lithothamnium calcareum, fato observado por Melo et al. (2006), onde avaliaram a solubildade in vitro de diversas fontes de cálcio (tabela II) e observaram que a farinha de algas calcáreas Lithothamnium 
Tabela II. Taxa de solubilidade in vitro de calcio de diversas fontes. (Rate of in vitro solubility of calcium from various sources).

\begin{tabular}{lc}
\hline Fontes de cálcio & Solubilidade\% \\
\hline Lithothamnium calcareum & $28,750^{\mathrm{a}}$ \\
Farinha de casca de ovos & $27,500^{\mathrm{ab}}$ \\
Farinha de ostras & $26,000^{\mathrm{bc}}$ \\
Fosfato bicálcico & $24,500^{\mathrm{c}}$ \\
Calcário & $19,375^{\mathrm{d}}$ \\
\hline
\end{tabular}

$\mathrm{CV}=3,89 \%$

Medias seguidas por letras diferentes na coluna diferem entre si pelo teste Tukey $(p<0,05)$. Adaptado de Melo et al. (2006).

calcareum apresentou valores maiores de solubilidade do que as demais fontes estudadas.

Sobre características de carcaça de frangos, não foram verificados efeito significativo do uso de farinha de algas calcáreas sobre a composição da carcaça de frangos de corte (Zanini et al., 2002). Anteriormente, Zanini et al. (2000b) observou que a utilização de farinha de algas calcáreas também não influenciou a deposição de gordura abdominal na carcaça de aves. Fialho et al. (1992), avaliando algumas fontes de suplementação de cálcio para suínos, relataram que as dietas tanto para crescimento como para terminação, podem ser suplementadas com cálcio provenientes do calcário calcítico, farinha de ostras, gesso ou Lithothamnium calcareum.

\section{AUTILIZAÇÃO NA ALIMENTAÇÃODE OUTRASESPÉCIES}

De acordo com Dutra et al. (1989), a utilização da alga calcária em rações de escargot Helix aspersa, pode ser utilizada como fonte de cálcio com efeitos significativos no crescimento do mesmo, quando comparado com calcário e farinha de ostras. Esses animais em crescimento demandam uma grande quantidade de cálcio para a formação e crescimento da concha, e o Lithothamnium calcareum foi capaz de suprir estas necessidades significativamente superior as demais fontes, sendo provável pela sua maior solubilidade em relação as demais

Em pesquisa com ratos, Assoumani (1997) relatou que a farinha de algas calcáreas apresentou vantagens em relação ao calcário no crescimento do osso fêmur e na biodisponibilidade de cálcio, sugerindo que provavelmente a concentração de magnésio e a porosidade da alga seriam os responsáveis por estas diferenças, já que a farinha de algas apresenta porosidade $(>40 \%)$ que propicia maior superfície específica de atuação.

\section{AUTILIZAÇÃONA ALIMENTAÇÃODE RUMINANTES}

Utilizando a farinha de algas marinhas como suplemento para vacas leiteiras, Melo et al. (2004a) estudaram o efeito de diferentes doses e concluíram que $50 \mathrm{~g} /$ animal/dia promoveram aumento da produção e do teor de gordura no leite, assim como o teor de cálcio e magnésio no sangue dos animais. Se desconhece a relação da farinha de algas com o aumento na produção e do teor de gordura no leite, porém o aumento do teor de cálcio e magnésio no sangue dos animais pode estar relacionado à maior biodisponibilidade destes nutrientes.

Melo et al. (2004b), relataram que para bovinos de corte, a utilização de $10 \%$ de farinha de algas calcáreas em substituição à mistura mineral comercial, promoveu aumento de $26 \%$ no ganho de peso dos animais, fato também observado por Souza (2002), que avaliou o uso de farinha de algas calcáreas na suplementação mineral de bovinos de corte e observou um ganho de peso $23 \%$ superior em relação aos animais que não receberam o suplemento, concluindo que o ganho de peso de um único dia é mais do que suficiente para pagar o 


\section{UTILIZAÇÃO DA FARINHA DE ALGAS CALCÁREAS NA ALIMENTAÇÃO ANIMAL}

suplemento usado durante o mês. Estes resultados de melhora no ganho de peso podem estar relacionados com o aumento da digestibilidade aparente da proteína bruta de forragens, fato também observado por Orsine et al. (1989) e Melo et al. (2002) os quais relataram que a adição da farinha de algas calcáreas na dieta de bovinos melhorou a qualidade e a produção de leite, promoveu aumento no ganho de peso e melhorou a digestibilidade aparente da proteína bruta de forragens de baixa qualidade.

Em dietas com altos niveis de concentrado TMR (Total Mixed Rations) Cruywagen et al. (2004), observaram que a inclusão de Lithothamnium calcareum na dieta como tamponante aumentou o $\mathrm{pH}$ ruminal, e que a melhor dose para otimizar a produção de leite foi de 80 gramas por dia do tamponante na dieta.

Corroborando com estes resultados, Montañez-Valdez et al. (2007), relataram que a inclusão de Lithothamnium calcareum

\section{BIBLIOGRAFIA}

Airhart, J.C., S.J. Taylor e K.W. Purser. 2002. The bioavailability in chicks of calcium in a product derived from calcified seaweed (Marigro). Disponível em: www.poultryscience.org/meet/ spss/spss2.pdf. Acesso em 15/07/2008.

Algarea. 2008. Disponível em: http://www. algarea.com.br/produto/granulado.htm. Acesso em 15/07/2008.

Assoumani, M.B. 1997. Aquamin, a natural calcium supplement derived from seaweed. Agro Food Industry Hi-Tech (September/October): 45-47.

Berberian, A. and O. Lenci. 1983. Emprego do suplemento mineral de alga LC-325 como nutriente de cavalos. Palestra proferida na semana do cavalo. Brasília, DF.

Bosence, D. 1976. Ecological studies on two unattached coralline algae from western Ireland. Paleontology, 19: 365-395.

Cabioch. J. 1970. Le maerl des côtes de Bretagne et le probleme de sa survie. Pen ar Bed, 7: 421429.

Couto, H.P., V.L.H. Nery, J.B. Fonseca, J. Chiquieri, L.C.R. Carneiro e C.T. Lombardi. 2008. Fontes como tamponante em dietas com $70 \%$ de concentrado, aumentou o $\mathrm{pH}$ ruminal e aumentou o desenvolvimento de protozoários ruminais e não prejudicou a digestibildade in situ da matéria seca e do FDN.

\section{CONSIDERAÇÕESFINAIS}

A utilização da farinha de algas calcáreas (Lithothamnium calcareum) na alimentação animal pode não ser recente, porém ainda necessita de diversas pesquisas para avaliarem os seus verdadeiros potenciais e níveis de utilização deste suplemento, pois ainda é muito incipiente de resultados sobre os efeitos desta suplementação em ambas as espécies. Sabemos que a farinha de algas calcáreas apresentam diversas características que podem ser interessantes e importantes à nível de nutrição animal, e o incentivo de mais pesquisas relacionadas a este assunto são essenciais para o desenvolvimento e conhecimento deste assunto.

alternativas de cálcio e fósforo para poedeiras comerciais. Rev. Bras. Zootecn., 37: 14191423.

Cruywagen, C.W., J. P. Swiegers, S.J. Taylor e E. Coetzee. 2004. The effect of acid buf in dairy cow diets on production response and rumen parameters. Disponível em: http://www.fass. org/2004/abstracts/39.PDF. Acesso em 09/06/ 2009.

Dias, G.T.M. 2000. Granulados bioclásticos - algas calcárias. Braz. J. Geophys., 18: 307-318.

Dutra, A.V.G., J.C.F. Aguiar, N.L. Antúnea e D.C. Pessoa.1989. Utilización del concentrado de alga calcárea (Lithothaminum sp.) en la ración de Helix aspersa Muller, 1774 (Gastropoda, helicidae). Rev. Cubana Alimentación y Nutrición, 3: 429-441.

Fassani, E. J., A.G. Bertechini, R.K. Kato, E.T. Fialho e A. Geraldo. 2004. Composição e solubilidade in vitro de calcários calcíticos de Minas Gerais. Ciênc. Agrotec., 28: 913-918.

Fialho, E.T., H.P. Barbosa, C. Bellaver, P.C. Gomes e W.B. Junior.1992. Avaliação nutricional de 


\section{MELO E MOURA}

algumas fontes de suplementação de cálcio para suínos. Biodisponibilidade e desempenho. Rev. Bras. Zootecn, 21: 891-905.

Gomes, A.S., J.J.C. Palma e C.G. Silva. 2000. Causas e conseqüências do impacto ambiental da exploração dos recursos minerais marinhos. Braz. J. Geophys., 18: 447-454.

Langwinski, D. e H. Ospina. 2001. A nutrição de ruminantes e os complexos orgânicos de minerais. Tortuga Companhia Zootécnica Agrária. Porto Alegre. 52 p.

Lenci, O. and A. Berberian. 1984. Contribuição ao estudo das consolidações de fraturas ósseas no PSI através do concentrado integral de alga LC-325. Relatório de pesquisa do Jockey Club -São Paulo. Brasil.

Macari, M., R.L. Furlan e L. Gonzales. 2002. Fisiologia aviária aplicada a frangos de corte. FUNEP/UNESP. Jaboticabal. p. 375.

Melo, P.C., A.B. Rezende e M.W.R. Souza. 2004a. Efeitos de doses do Lithothamnium $\mathrm{sp}$ na produção leiteira. Disponível em: www. calmarmineracao.cm.br/calmar/farinha2.htm. Acesso em 4/09/2004.

Melo, P.C., C.A.R. Franco e A.R. Franco. 2004b. Uso de farinha de algas marinhas (Lithothamnium $\mathrm{sp)}$ na suplementação mineral de bovinos de corte. Disponível em: www.calmarmineracao. com.br/calmar/farinha1.htm. Acesso em 4/09/ 2004.

Melo, P.C., F.C. Silva e M.W.R. Souza. 2002. Efeito de doses do SUMINAL $\circledast$ na produção leiteira. XII Congresso Brasileiro de Zootecnia e IV Congresso Internacional de Zootecnia. Rio de Janeiro, Anais.... Rio de Janeiro-RJ.

Melo, T.V. 2006. Utilização de farinha de algas marinhas (Lithothamnium calcareum) e de fosfato monoamônio em rações para codornas japonesas em postura criadas sob condições de calor. Dissertação (Mestrado em Produção Animal), Universidade Estadual do norte Fluminense.UENF. Campos do Goytacazes-RJ. Brasil. 56 p.

Melo, T.V., P.P. Mendonça, A.M.A. Moura, C.T. Lombardi, R.A. Ferreira e V.L.H. Nery. 2006. Solubilidad in vitro de algunas fuentes de cálcio utilizadas em alimentacion animal. Arch. Zootec., 55: 297-300.

Melo, T.V., R.A. Ferreira, V.C. Oliveira, J.B.A. Carneiro, A.M.A. Moura, C.S. Silva e V.L.H.
Nery. 2008a. Calidad del huevo de codornices utilizando harina de algas marinas y fosfato monoamónico. Arch. Zootec., 57: 313-319.

Melo, T.V., R.A. Ferreira, J.B.A. Carneiro, V.C. Oliveira, A.M.A. Moura, C.S. Silva e V.L.H. Nery. 2008b. Rendimiento de codornices japonesas utilizando harina de algas marinas y fosfato monoamónico. Arch. Zootec., 57: 381-384.

Montañez-Valdez, O.D., E.O. Garcia-Flores, J.R. Barcena-Gama, S.S. Gonzalez-Muñoz, M.E. Ortega-Cerrilla, J.G. Peralta-Ortiz e J.H. Avellaneda-Cevallos. 2007. Effect of two buffers on nutrient digestibilities and ruminal fermentation in Holstein steers. Disponível em: http://adsa.asas.org/meetings/2007/abstracts/ 0545.PDF. Acesso em 09/06/2009.

Muniz, E.B., A.M.V. Arruda, E.J. Fassani, A.S. Teixeira e E.S. Pereira. 2007. Avaliação de fontes de cálcio para frangos de corte. Caatinga, 20: 5-14.

Orsine, G.F., C.P. Costa, B. Oliveira, D.O. Rodrigues e C.R. Oliveira. 1989. Efeito da fonte de cálcio (calcário vs Lithothamnium calcareum) na digestibilidade aparente do feno de capim Brachiaria decumbens Stach cv. Basiliski. Anais Esc. Agron. e Vet., 19: 49-58.

Pelicia, K., E. A. Garcia, M. R. Scherer, C. Mori, J.A. Dalanezi, A.B.G. Faitarone, E.S.P.B. Saldanha, C.C Pizzolante, A.M. Brito e D. Berto. 2006. Efeito da combinação de fontes de cálcio sobre o desempenho e qualidade de ovos de poedeiras comerciais. In: 43a Reunião Anual da SBZ, 2006. João Pessoa-PB.

Perali, C., M. Aranovich, M.W. Santos, S. Aranovich, D.M.F. Costa, G.M. Silva e V.F. Rocha. 2003. Efeito de diferentes níveis de adição do Suminal® sobre a produção e peso de ovos de codornas alimentadas com concentrados. 40 Reunião Anual da Sociedade Brasileira de Zootecnia. Santa Maria, Anais (CD) .... Sociedade Brasileira de Zootecnia. Santa Maria.

Pope, H.R., C.M. Owens, L.C. Cavitt, J.L. Emmert e S.J. Taylor. 2002. Efficacy of marigro in supporting growth, carcass yield and meat quality of broilers. $91^{\text {st }}$ Annual Meeting Abstracts. The Southern Poultry Science Society. Poscal 80 (Suppl. 1). p. 25.

Souza, E.F. 2002. Relatório sobre experimento com o uso de farinha de algas marinhas na suplementação mineral de bovinos de corte. 


\section{UTILIZAÇÃO DAFARINHA DE ALGAS CALCÁREAS NA ALIMENTAÇÃO ANIMAL}

Disponível em: http://www.naturalrural. com.br/conteudo/experimentos_Ic300_ suplementacao_bovinos.pdf. Acesso em 15/ 07/2008.

Spears, J.W. 1996. Organic trace minerals in ruminant nutrition. Anim. Feed Sci. Techn., 58: 151-163.

Waldroup, P.W. 1996. Bioassays remain necessary to estimate phosphorus, calcium bioavailability. Feedstuffs, 68: 13-20.

Zanini, S.F., M.A.G. Carvalho, G. Colnago e R. Quilula. 2000a. Uso de farinha de algas como fonte de cálcio na ração de frangos de corte. In: Reunião Anual da Sociedade Brasileira de Zoo- tecnia XXXVII. Viçosa, MG. Anais... Sociedade Brasileira de Zootecnia. Viçosa-MG.

Zanini, S.F., M.A.G. Carvalho, G. Colnago, R. Quilula e L.F. Miranda. 2000b. Uso de farinha de algas na ração sobre o teor de gordura abdominal de frangos de corte. In: Seminário de Extensão e Pesquisa do ES, (2) Vitória. Anais... UFES. Vitória. p. 79.

Zanini, S.F., M.A.G. Carvalho, G. Colnago, R. Quilula, A. M. N. Korres, K. S. Santos, C. O. Silva Júnior, A.C. Soares e F. Fava. 2002. Composição da carcaça de frangos de corte submetidos a dieta com farinha de algas. Rev. Centro Univ. Vila Velha (ES), 3 (janeiro/julho): 45. 